\title{
Erratum to: On the Number of Invariants in the Strain Energy Density of an Anisotropic Nonlinear Elastic Material with Two Material Symmetry Directions
}

\author{
Adair Roberto Aguiar ${ }^{1,2}$ - Gabriel Lopes da Rocha ${ }^{2}$
}

Published online: 3 July 2017

(C) Springer Science+Business Media B.V. 2017

\section{Erratum to: J Elast (2017)}

DOI 10.1007/s10659-017-9642-5

This erratum concerns a correction in the formulae (19) and (23) of the original article. It also concerns the addition of Remark 5, which contains two relations obtained from (19) and their correspondence to two syzygys found by Shariff and Bustamante [1], which also appear in [2]. These works were not known to the authors at the time of publication of the article.

The correct formulae in (19) are

$$
\begin{aligned}
b_{1}^{2}= & J_{10}, \quad C_{11}=I_{5}, \quad b_{1} b_{2} C_{12}=J_{11}-I_{5} J_{10}, \\
C_{22}= & \left(I_{8}+I_{5} J_{10}-2 J_{11}\right) /\left(1-J_{10}\right), \quad C_{33}=J_{1}-\left(I_{8}+I_{5}-2 J_{11}\right) /\left(1-J_{10}\right), \\
C_{13}^{2}= & J_{15} /\left(1-J_{10}\right), \quad C_{12}^{2}=I_{6}-I_{5}^{2}-J_{15} /\left(1-J_{10}\right), \\
C_{23}^{2}= & -\left(4 J_{11}^{2}+J_{10}^{2} J_{2}+J_{10}^{2} I_{6}-2 J_{10} J_{2}+J_{10} I_{5}^{2}-2 J_{10} I_{6}+J_{2}+I_{6}+I_{5}\left(J_{10}+1\right) I_{8}\right. \\
& \left.+I_{8}^{2}+J_{1}\left(J_{10}-1\right)\left(-2 J_{11}+I_{5}+I_{8}\right)-2 J_{11}\left(J_{10} I_{5}+I_{5}+2 I_{8}\right)\right) /\left(J_{10}-1\right)^{2},
\end{aligned}
$$

The online version of the original article can be found under doi:10.1007/s10659-017-9642-5.

$凶$ A.R. Aguiar

aguiarar@sc.usp.br

1 Department of Structural Engineering, São Carlos School of Engineering, University of São Paulo, São Carlos, SP, Brazil

2 Programa de Pós-Graduação Interunidades em Bioengenharia - EESC/FMRP/IQSC,

University of São Paulo, São Carlos, SP, Brazil 


$$
\begin{aligned}
C_{12} C_{13} C_{23}= & \left(J_{10}^{2} J_{3}+J_{10} J_{15} I_{5}-J_{10}^{2} J_{2} I_{5}-J_{10}^{2} I_{5} I_{6}-2 J_{10} J_{3}+J_{15} I_{5}\right. \\
& +2 J_{10} J_{2} I_{5}-J_{10} I_{5}^{3}+3 J_{10} I_{5} I_{6}+J_{3}-J_{2} I_{5}+I_{5}^{3}-2 I_{5} I_{6} \\
& +J_{1}\left(J_{10}-1\right)\left(J_{15}+I_{6}\left(J_{10}-1\right)\right)-2 J_{11}\left(2 J_{15}+\left(I_{5}^{2}-I_{6}\right)\left(-J_{10}+1\right)\right) \\
& \left.+\left(2 J_{15}+\left(I_{5}^{2}-I_{6}\right)\left(-J_{10}+1\right)\right) I_{8}\right) /\left(2\left(J_{10}-1\right)^{2}\right), \\
b_{1} b_{2} C_{13} C_{23}= & \left(J_{10}^{2}\left(J_{2}+I_{6}\right)-J_{10}\left(J_{15}+2 J_{11} I_{5}+2 J_{2}-I_{5}^{2}+I_{6}-I_{5} I_{8}\right.\right. \\
& \left.-J_{1}\left(-2 J_{11}+I_{5}+I_{8}\right)+I_{9}\right)+\left(4 J_{11}^{2}+2 J_{11}\left(J_{1}-2 I_{5}-I_{8}\right)\right. \\
& \left.\left.+\left(J_{15}+J_{2}-\left(J_{1}-I_{5}\right)\left(I_{5}+I_{8}\right)+I_{9}\right)\right)\right) /\left(2\left(1-J_{10}\right)\right) .
\end{aligned}
$$

Substituting the expressions of $b_{1} b_{2} C_{12}, b_{1} b_{2} C_{13} C_{23}$, and $C_{12} C_{13} C_{23}$, given in (19), into $b_{1} b_{2} C_{12} b_{1} b_{2} C_{13} C_{23}=\left(b_{1} b_{2}\right)^{2} C_{12} C_{13} C_{23}$, we obtain the correct expression for $J_{3}$, which is given by

$$
\begin{aligned}
J_{3}= & \left(J_{11}^{3}+J_{1} J_{10}\left(-J_{11}^{2}-J_{10} I_{5} I_{8}+J_{11}\left(I_{5}+I_{8}\right)\right)\right. \\
& +J_{10} J_{11}\left(\left(-1+J_{10}\right) J_{2}-I_{6}-I_{5} I_{8}-I_{9}\right) \\
& \left.+J_{10}^{2}\left(I_{6} I_{8}+I_{5} I_{9}\right)\right) /\left(J_{10}^{2}\left(-1+J_{10}\right)\right) .
\end{aligned}
$$

Remark 5 Below we use both (22) and (23) in all the calculations and consider that $J_{10}$ does not vanish. Substituting the expressions of $C_{13}^{2}, C_{23}^{2}$, and $b_{1} b_{2} C_{13} C_{23}$, given in (19), into $A_{1} \triangleq\left(b_{1} b_{2}\right)^{2} C_{13}^{2} C_{23}^{2}-\left(b_{1} b_{2} C_{13} C_{23}\right)^{2}$, we obtain

$$
\begin{aligned}
A_{1} \triangleq & \left(4 ( J _ { 1 1 } ^ { 2 } - 2 J _ { 1 0 } J _ { 1 1 } I _ { 5 } + J _ { 1 0 } ( I _ { 5 } ^ { 2 } + ( - 1 + J _ { 1 0 } ) I _ { 6 } ) ) \left(4 J_{11}^{2}+J_{2}+I_{6}\right.\right. \\
& +J_{10}\left(\left(-2+J_{10}\right) J_{2}+I_{5}^{2}+\left(-2+J_{10}\right) I_{6}\right)-J_{1}\left(-1+J_{10}\right)\left(2 J_{11}-I_{5}-I_{8}\right) \\
& \left.+\left(1+J_{10}\right) I_{5} I_{8}+I_{8}^{2}-2 J_{11}\left(I_{5}+J_{10} I_{5}+2 I_{8}\right)\right)-\left(\left(\left(-1+5 J_{10}\right) J_{11}^{2}\right.\right. \\
& -J_{1}\left(-1+J_{10}\right) J_{10}\left(2 J_{11}-I_{5}-I_{8}\right)-2 J_{10} J_{11}\left(I_{5}+2 J_{10} I_{5}+I_{8}\right) \\
& +J_{10}\left(\left(-1+J_{10}\right)^{2} J_{2}+I_{6}+2 J_{10}^{2} I_{6}+I_{5} I_{8}\right. \\
& \left.\left.\left.\left.+J_{10}\left(-3 I_{6}+I_{5}\left(2 I_{5}+I_{8}\right)-I_{9}\right)+I_{9}\right)\right)^{2}\right) / J_{10}^{2}\right) /\left(4\left(-1+J_{10}\right)^{2}\right) .
\end{aligned}
$$

Similarly, substituting the expressions of $C_{12}^{2}, C_{13}^{2}, C_{23}^{2}$, and $C_{12} C_{13} C_{23}$, given in (19), into $A_{2} \triangleq\left(C_{12} C_{13} C_{23}\right)^{2}-C_{12}^{2} C_{13}^{2} C_{23}^{2}$, we obtain

$$
\begin{aligned}
A_{2}= & \left(J_{11}-J_{10} I_{5}\right)^{2}\left(\left(-1+9 J_{10}\right) J_{11}^{4}-4 J_{10} J_{11}^{3}\left(I_{5}+I_{8}\right)\right. \\
& +J_{1}^{2}\left(-1+J_{10}\right) J_{10}^{2}\left(-2 J_{11}+I_{5}+I_{8}\right)^{2}+2 J_{10} J_{11}^{2}\left(\left(-1+J_{10}\right)\left(-1+3 J_{10}\right) J_{2}\right. \\
& \left.-\left(-1+5 J_{10}\right)\left(I_{6}+I_{5} I_{8}+I_{9}\right)\right)+4 J_{10}^{2} J_{11}\left(I_{5}^{2} I_{8}-\left(-1+J_{10}\right) J_{2}\left(I_{5}+I_{8}\right)\right. \\
& \left.+I_{8}\left(I_{6}+2 J_{10} I_{6}+I_{9}\right)+I_{5}\left(I_{6}+I_{8}^{2}+I_{9}+2 J_{10} I_{9}\right)\right)+J_{10}^{2}\left(\left(-1+J_{10}\right)^{3} J_{2}^{2}\right. \\
& +\left(-1+J_{10}\right) I_{6}^{2}+\left(-1+J_{10}\right) I_{5}^{2} I_{8}^{2}-2 I_{5}\left(I_{8}+J_{10}\left(2 I_{5}+I_{8}\right)\right) I_{9}+\left(-1+J_{10}\right) I_{9}^{2} \\
& -2\left(-1+J_{10}\right) J_{2}\left(\left(-1+J_{10}\right) I_{6}-\left(1+J_{10}\right) I_{5} I_{8}+\left(-1+J_{10}\right) I_{9}\right)
\end{aligned}
$$




$$
\begin{aligned}
& \left.-2 I_{6}\left(I_{8}\left(I_{5}+J_{10} I_{5}+2 J_{10} I_{8}\right)+I_{9}+J_{10}\left(-3+2 J_{10}\right) I_{9}\right)\right) \\
& -2 J_{1} J_{10}\left(2 J_{11}-I_{5}-I_{8}\right)\left(\left(-1+3 J_{10}\right) J_{11}^{2}-2 J_{10} J_{11}\left(I_{5}+I_{8}\right)+J_{10}\left(\left(-1+J_{10}\right)^{2} J_{2}\right.\right. \\
& \left.\left.\left.+I_{6}+I_{5} I_{8}+I_{9}-J_{10}\left(I_{6}-I_{5} I_{8}+I_{9}\right)\right)\right)\right) /\left(4\left(-1+J_{10}\right)^{3} J_{10}^{4}\right) .
\end{aligned}
$$

By definition, however, both $A_{1}$ and $A_{2}$ are related to each other through the expression $\left(J_{11}-J_{10} I_{5}\right)^{2} A_{1}=-J_{10}^{2}\left(-1+J_{10}\right)^{2} A_{2}$, where we have used the expressions of $b_{1}^{2}$ from (19) and $C_{12}$ in (21). It is then clear that only one relation between the invariants can be obtained from both (24) and (25).

On the other hand, Shariff and Bustamante [1] have obtained three relations, given by (30), (34), and (39), between the invariants in the set (3) of their work. These invariants correspond to the invariants listed in (7) of our article. The authors claim that these three relations can be used to reduce the number of invariants in (3) of their work from ten to seven, or, from nine to six if their ninth invariant, $I_{9}$, is known. We show below that these relations can only give two independent invariants. The third invariant is precisely $J_{3}$, given by (23) above.

(a) Their relation (30) corresponds to the polynomial relation (8) in our article, yielding, for instance, $(\mathbf{a} \cdot \mathbf{b})\left(\mathbf{a} \cdot \mathbf{C}^{2} \mathbf{b}\right)$ in terms of the other invariants.

(b) Their relation (34) is not correct. Its right-hand side should be multiplied by $\left(1-I_{9}\right)^{2} I_{9}^{2}$ in their notation. Passing the correct right-hand side to the left-hand side of the relation (34) and denoting by $B_{1}$ the resulting left-hand side, we find that $B_{1}=A_{1} R_{1}$, where $A_{1}$ is given by the expression (24) above and $R_{1}$ is a polynomial of the invariants, which, in general, does not vanish. This means that both $A_{1}$ and $B_{1}$ yield the same syzygy.

(c) Passing the right-hand side of their relation (39) to the left-hand side and denoting by $B_{2}$ the resulting left-hand side, we find that $B_{2}=4 A_{2}$, where $A_{2}$ is given by the expression (25) above. We also find that $B_{1}$ and $B_{2}$ are related by the expression

$$
-4\left(J_{11}-J_{10} I_{5}\right)^{2} B_{1} /\left(J_{10}^{2}\left(-1+J_{10}\right)^{2}\right)=B_{2} R_{1},
$$

where $R_{1}$ is the polynomial that appears in Item b) above. By definition, both sides of (26) must be zero. Since, in general, both $J_{11}-J_{10} I_{5}$ and $R_{1}$ do not vanish, we must have that an invariant that satisfies $B_{1}=0$ must also satisfy $B_{2}=0$ and conversely. Therefore, $B_{1}$ and $B_{2}$ yield one independent invariant, provided that $J_{3}$ is known from (23) above.

To conclude this remark, we still need a set of seven invariants, given by $\left\{J_{1}, J_{2}, I_{5}, I_{6}, I_{8}\right.$, $\left.I_{9}, J_{11}\right\}$, to characterize the strain energy function of an anisotropic elastic material with two non-orthogonal symmetry directions. Similarly to the case of orthogonal directions, these invariants satisfy only one syzygy, given by either $A_{1}=0$ or $A_{2}=0$. Recall from above that Shariff and Bustamante [1] claim the existence of two syzygys, given by both $B_{1}=0$ and $B_{2}=0$.

Acknowledgements The authors gratefully acknowledge the financial support from the Coordination for the Improvement of Higher Education Personnel (CAPES) and the National Council for Scientific and Technological Development (CNPq), under grant 444896/2014-7. They are also grateful to Jose Merodio for bringing the references below to their attention. 


\section{References}

1. Shariff, M.H.B.M., Bustamante, R.: On the independence of strain invariants of two preferred direction nonlinear elasticity. Int. J. Eng. Sci. 97, 18-25 (2015)

2. Shariff, M.H.B.M.: The number of independent invariants of an $n$-preferred direction anisotropic solid. Mathematics and Mechanics of Solids, 1-8. doi:10.1177/1081286516653196 